PROCEEDINGS OF THE

AMERICAN MATHEMATICAL SOCIETY

Volume 133, Number 3, Pages 677-679

S 0002-9939(04)07643-9

Article electronically published on August 4, 2004

\title{
EQUIVALENCE OF THE NASH CONJECTURE FOR PRIMITIVE AND SANDWICHED SINGULARITIES
}

\author{
JESÚS FERNÁNDEZ-SÁNCHEZ
}

(Communicated by Michael Stillman)

\begin{abstract}
We show that in order to prove the Nash Conjecture for sandwiched singularities it is enough to prove it for primitive singularities.
\end{abstract}

Let $(X, Q)$ be a normal surface singularity and $f: \widehat{S} \longrightarrow(X, Q)$ its minimal resolution. An arc (an arc of order $i)$ on $(X, Q)$ is a local $\mathbb{C}$-morphism $\mathcal{O}_{X, Q} \longrightarrow$ $\mathbb{C}[[t]]$ (a local $\mathbb{C}$-morphism $\mathcal{O}_{X, Q} \longrightarrow \mathbb{C}[[t]] /\left(t^{i+1}\right)$ ). If $\left\{E_{u}\right\}_{u \in T_{Q}}$ are the irreducible exceptional components of $\widehat{S}$, let $\mathcal{F}_{u}^{Q}$ denote the set of arcs on $(X, Q)$ such that the lifted arc $\widetilde{\varphi}$ on $\widehat{S}$ intersects $E_{u}$. Let $\mathcal{H}\left(\mathcal{H}_{i}\right.$ for $\left.i \geq 0\right)$ be the space of arcs (arcs of order $i$ ) on $(X, Q)$. Define $\operatorname{Tr}(i)$ to be the space of $i$-truncations of $\operatorname{arcs}$ on $(X, Q)$, that is $\operatorname{Tr}(i)=\rho_{i}(\mathcal{H})$, where $\rho_{i}: \mathcal{H} \longrightarrow \mathcal{H}_{i}$ is the morphism induced by the projection $\mathbb{C}[[t]] \longrightarrow \mathbb{C}[[t]] /\left(t^{i+1}\right)$. Then, if $\mathcal{F}_{u}^{Q}(i)=\rho_{i}\left(\mathcal{F}_{u}^{Q}\right)$, the minimal resolution $f: \widehat{S} \longrightarrow(S, 0)$ induces a decomposition of the space of $i$-truncations $\operatorname{Tr}(i)=\bigcup_{u \in T_{Q}} \mathcal{F}_{u}^{Q}(i)$ and, by taking the Zariski closure in $\mathcal{H}_{i}$,

$$
\overline{\operatorname{Tr}(i)}=\bigcup_{u \in T_{Q}} \overline{\mathcal{F}_{u}^{Q}(i)}
$$

The Nash Conjecture says that for $i \gg 0$, this is just the decomposition of $\overline{\operatorname{Tr}(i)}$ into its irreducible components (see [5]).

A normal surface singularity $(X, Q)$ is said to be sandwiched if it dominates birationally a non-singular surface. They arise when a complete $\mathbf{m}$-primary ideal in a local regular $\mathbb{C}$-algebra $R$ of dimension two is blown up. A sandwiched singularity is said to be primitive if it can be obtained by blowing up a simple ideal, that is, a complete irreducible ideal of $R$ ([7] II.3). It is known that any sandwiched singularity is the birational join of some primitive singularities (7] Corollary II.1.5). In this note, we prove that the Nash Conjecture for sandwiched singularities and for primitive singularities are equivalent.

First, we need a lemma.

Lemma 1. Let $\left(X_{1}, Q_{1}\right)$ be a rational surface singularity, $g: X \longrightarrow\left(X_{1}, Q_{1}\right)$ a birational dominant morphism and $E_{p}$ an exceptional component of $Q$ such that $E_{p}$ also appears in the minimal resolution of $Q_{1}$ modulo birational equivalence (see

Received by the editors August 27, 2003 and, in revised form, November 1, 2003.

2000 Mathematics Subject Classification. Primary 14B05, 14E15, 32B10.

Key words and phrases. Sandwiched singularity, arc, infinitely near point.

The author was partially supported by Generalitat de Catalunya 2001SGR00071 and EAGER, European Union contract HPRN-CT-2000-00099.

(C)2004 American Mathematical Society Reverts to public domain 28 years from publication 
Definition 2.1 of [4]). Keeping the notation as above, assume that for some $i>0$, $\overline{\mathcal{F}_{p}^{Q}(i)} \subset \overline{\mathcal{F}_{q}^{Q}(i)}$ and that the projection by $g$ of any element of $\mathcal{F}_{q}^{Q}(i)$ is in $\mathcal{F}_{u}^{Q_{1}}(i)$, for some $u \in T_{Q_{1}}$. Then,

$$
\overline{\mathcal{F}_{p}^{Q_{1}}(i)} \subset \overline{\mathcal{F}_{u}^{Q_{1}}(i)} .
$$

Proof. The morphism $g: X \longrightarrow\left(X_{1}, Q_{1}\right)$ is the blow-up of a complete ideal $J=$ $\left(g_{1}, \ldots, g_{m}\right) \subset A=\mathcal{O}_{X_{1}, Q_{1}}$, and we may assume that $Q \in U_{0}$, where $U_{0}$ is an affine open set of $X$ of the form $\operatorname{Spec} A\left[g_{1} / g_{0}, \ldots, g_{m} / g_{0}\right] \subset \mathbb{A}_{A}^{m}$. Now, if $\operatorname{Spec}(A) \subset \mathbb{A}_{\mathbb{C}}^{n}$, any arc $\gamma$ on $\left(X_{1}, Q_{1}\right)$ is written in the form $\gamma=\left(x_{1}, \ldots, x_{n}\right), x_{k} \in \mathbb{C}[[t]]$. Thus, the lifting $\widetilde{\gamma}$ of $\gamma$ on $X$ is given by

$$
\left(x_{1}(t), \ldots, x_{n}(t), \overline{g_{1}}(t) / \overline{g_{0}}(t), \ldots, \overline{g_{m}}(t) / \overline{g_{0}}(t)\right)
$$

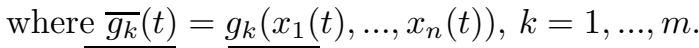

If $\overline{\mathcal{F}_{p}^{Q}(i)} \subset \overline{\mathcal{F}_{q}^{Q}(i)}$, the $i$-truncation of any arc of $\mathcal{F}_{p}^{Q}$ can be approximated by the $i$-truncations of arcs of $\mathcal{F}_{q}^{Q}$. By taking the projections of these $i$-truncations on $(X, Q)$, we see that $\mathcal{F}_{p}^{Q_{1}}(i) \subset \overline{g_{*}\left(\mathcal{F}_{q}^{Q}(i)\right)} \subset \overline{\mathcal{F}_{u}^{Q_{1}}(i)}$ and hence, $\overline{\mathcal{F}_{p}^{Q_{1}}(i)} \subset \overline{\mathcal{F}_{u}^{Q_{1}}(i)}$.

Remark 1. A similar result has been proved independently by Camille Plénat [6].

From now on, assume that $(X, Q)$ is a sandwiched singularity and that $X=$ $B l_{I}(R)$ is the surface obtained by blowing up a complete $\mathbf{m}$-primary ideal $I$ in a regular local two-dimensional $\mathbb{C}$-algebra $R$. Assume also that $I$ satisfies the conditions of Corollary 1.14 of [7. In particular, if

$$
I=\prod_{j=1}^{N} I_{j}
$$

is the factorization of $I$ into simple complete ideals, we have that $I_{j} \neq I_{k}$ for $j \neq k$, and for $j=1, \ldots, N$ the surface $X_{j}=B l_{I_{j}}(R)$ has only one singularity, that will be denoted by $Q_{j}$.

Following the notation introduced in [3], we denote by $\mathcal{K}=B P(I)$ and $\mathcal{K}_{j}=$ $B P\left(I_{j}\right)$ the clusters of base points of $I$ and the simple ideals $I_{j}$, for $j=1, \ldots, N$. We also denote by $S_{K}$ and $S_{j}$ the surfaces obtained by blowing up all the points of the clusters $\mathcal{K}$ and $\mathcal{K}_{j}$. The reader is referred to [2] for the connection between sandwiched singularities and clusters, and to chapters 3 and 4 of [1] for conventions and definitions about clusters.

It is known that the morphisms $f: S_{K} \longrightarrow X$ and $f_{j}: S_{j} \longrightarrow X_{j}$ induced by the universal property of the blowing up are the minimal resolutions of $X$ and $X_{j}, j=1, \ldots, N$ respectively. Moreover, $X$ and $S_{K}$ are the birational join of the surfaces $X_{j}$ and $S_{j}$ for $j=1, \ldots, N(7]$ Proposition 3.6). If we write $\alpha_{j}: X \longrightarrow X_{j}$ for the blow up of $I \mathcal{O}_{X_{j}}$ in $X_{j}$ and $\sigma_{j}: S_{K} \longrightarrow S_{j}$ for the induced morphism, we have commutative diagrams of birational morphisms:

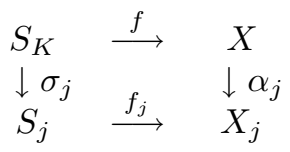

By abuse of notation, we will write $\left\{E_{u}\right\}_{u \in T_{Q_{j}}}$ for the exceptional components of $f_{j}: S_{j} \longrightarrow X_{j}$. 
Theorem 2. Let $p, q \in T_{Q}, p \neq q$, be such that $\overline{\mathcal{F}_{p}^{Q}(i)} \subset \overline{\mathcal{F}_{q}^{Q}(i)}$. Then there exists some $j \in\{1, \ldots, N\}$ with $p \in T_{Q_{j}}$ and some $u \in T_{Q_{j}}, u \neq p$, such that $\overline{\mathcal{F}_{p}^{Q_{j}}(i)} \subset \overline{\mathcal{F}_{u}^{Q_{j}}(i)}$

Proof. Assume that $p, q \in T_{Q}$ are points such that $\overline{\mathcal{F}_{p}^{Q}(i)} \subset \overline{\mathcal{F}_{q}^{Q}(i)}$ for $i \gg 0$. By Theorem 3.4 of [3], we know that $q$ is not infinitely near to $p$. Let $\mathcal{K}_{j}$ be any irreducible subcluster of $\mathcal{K}$ containing $p$.

If $p$ is infinitely near to $q$, then $q$ is also in $\mathcal{K}_{j}$ and hence, the exceptional components $E_{p}$ and $E_{q}$ also appear in the minimal resolution $S_{j}$ of $Q_{j}$ modulo birational equivalence. By Lemma 1 applied to $\alpha_{j}: X \longrightarrow X_{j}$, we deduce that $\overline{\mathcal{F}_{p}^{Q_{j}}(i)} \subset \overline{\mathcal{F}_{q}^{Q_{j}}(i)}$

If $p$ is not infinitely near to $q$, let $u_{0}$ be the maximal point of $\mathcal{K}_{j}$ to which $q$ is infinitely near. Then the projection by $\alpha_{j}$ of any element in $\mathcal{F}_{q}^{Q}$ gives an arc $\gamma$ on $\left(X_{j}, Q_{j}\right)$ whose lifting to $S_{j}$ intersects (transversally or not) the exceptional component $E_{u_{0}}$ and therefore, $\gamma \in \mathcal{F}_{u_{0}}^{Q_{j}}$. Thus, the projection of any element of $\mathcal{F}_{q}^{Q}(i)$ is in $\mathcal{F}_{u_{0}}^{Q_{1}}(i)$ and by Lemma 1 again, we deduce that $\overline{\mathcal{F}_{p}^{Q_{j}}(i)} \subset \overline{\mathcal{F}_{u_{0}}^{Q_{j}}(i)}$.

In any case, we see that an inclusion of spaces of arcs on the sandwiched singularity $(X, Q)$ implies a non-trivial inclusion of some spaces of arcs on the primitive singularity $\left(X_{j}, Q_{j}\right)$. The claim follows.

As a direct consequence, we have the following corollary.

Corollary 3. If the Nash Conjecture is true for primitive singularities, then it is also true for sandwiched singularities.

Remark 3. Notice that if $(X, Q)$ is a primitive singularity and we have $\overline{\mathcal{F}_{p}^{Q}(i)} \subset$ $\overline{\mathcal{F}_{q}^{Q}(i)}$ for $p, q \in T_{Q}$ with $p \neq q$, then by 3.4 of [3], $p$ must be infinitely near to $q$ and moreover, $e_{O}\left(I_{p}\right)>e_{O}\left(I_{q}\right)$ where $e_{O}(J)$ is the minimum of the multiplicities of the curves defined by elements of the complete ideal $J$.

\section{REFERENCES}

[1] E. Casas-Alvero, Singularities of plane curves, volume 276 of London Math. Soc. Lecture Note Series. Cambridge University Press, 2000. MR1782072 (2003b:14035)

[2] J. Fernández-SÁnchez, On sandwiched singularities and complete ideals. J. Pure Appl. Algebra, 185: 165-175, 2003. MR2006424

[3] J. Fernández-SÁnchez, Nash families of smooth arcs on a sandwiched singularity. To appear in Math. Proc. Cambridge. Philos. Soc.

[4] S. Ishit, J. Kollár, The Nash problem on arc families of singularities. Preprint. math.AG/0207171. Duke Math. J., 120: 601-620, 2003. MR2030097

[5] J. NASH, Arc structure of singularities. Duke Math. J., 81:31-38, 1996. MR1381967(98f:14011)

[6] C. Plénat, A propos de la conjecture de Nash. Preprint. math.AG/0301358.

[7] M. SpIvakovsky, Sandwiched singularities and desingularization of surfaces by normalized transformations. Ann. of Math., 131:411-491, 1990. MR1053487(91e:14013)

Departament d’Àlgebra i Geometria, Universitat de Barcelona, Gran Via, 585, E08007, BarCelona, SPAin

E-mail address: jsan@cerber.mat.ub.es 